\title{
A Search for Large Twin Prime Pairs
}

\author{
By R. E. Crandall and M. A. Penk
}

\begin{abstract}
Two methods are discussed for finding large integers $m$ such that $\boldsymbol{m}-1$ and $m+1$ are both primes. Eight such mumbers $m$ of magnitudes 22, 22, 32, 64, $136,154,203$, and 303 digits are listed; together with primitive roots (for $m+1$ ) and Lucas-Lehmer parameters (for $m-1$ ). The Hardy-Littlewood twin prime conjecture is supported by a statistical test involving the generation of 249 twin prime pairs in the 50-to-54 digit region.
\end{abstract}

1. Asymptotic Estimates. We define a twin prime mean (TPM) to be any positive integer $m$ such that $(m-1, m+1)$ is a pair of primes. It is unknown whether $M(x)$, the number of TPMs not exceeding $x$, is bounded as $x$ increases. However, in $1919 \mathrm{~V}$. Brun [1], by showing in effect that for some positive constant $C$,

$$
M(x)<\frac{C x}{\log ^{2} x}(\log \log x)^{2}
$$

established that the sum of the reciprocals of all TPMs must converge. Twin prime pairs $(m-1, m+1)$ are, therefore, significantly sparser than the primes themselves, so the task of discovering large TPMs is both interesting and challenging.

For the computer search reported here, we have made practical use of certain asymptotic estimates concerning the distribution of TPMs. Evidently, if $m$ is a TPM, then for any prime $p<m-1$, we have $m^{2} \neq 1(\bmod p)$. In particular, if $m>4$, then $m \equiv 0(\bmod 6)$. We define a sieving set with sieve limit $x$ to be the set of all primes $p$ with $5 \leqslant p \leqslant x$; this set is denoted as $P_{x}$. Then a function $Q(x)$ is defined

$$
Q(x)=\prod_{p \in P_{x}}\left(1-\frac{2}{p}\right) \sim \frac{K}{-\log ^{2} x},
$$

where $K$ is a positive constant. For an integer $n \equiv 0(\bmod 6)$, much larger than $x$ but otherwise "random", we loosely interpret $Q(x)$ as the "probability" that $n^{2}-1$ is prime to all primes in $P_{x}$. In 1923 Hardy and Littlewood used functions similar to $Q$ to derive an heuristic estimate [2]

$$
M(z) \sim \frac{2 C_{2} z}{\log ^{2} z},
$$

where $C_{2}$ is a constant $0.66016181 \ldots$ related to $K$ and Euler's constant $\gamma$ by $C_{2}=$ $K e^{2 \gamma} / 4$. The Hardy-Littlewood estimate is apparently in good agreement with actual counts of TPMs [3], [4], [5].

Received March 31, 1978; revised June 15, 1978.

AMS (MOS) subject classifications (1970). Primary 10A25, $10 \mathrm{~A} 40$.

Key words and phrases. Prime, twin primes, Hardy-Littlewood conjecture.

(C) 1979 American Mathematical Society 
To test whether some $m \equiv 0(\bmod 6)$ is a TPM it is natural to first choose a sieve limit $x$ and test both $m+1$ and $m-1$ for divisors in $P_{x}$. In the absence of any such divisors we then apply Fermat tests, that is to determine whether $2^{m} \equiv 1(\bmod m+1)$ and $2^{m-2} \equiv 1(\bmod m-1)$ as must both be true if $m \pm 1$ are both primes. In practice, these tests are virtually conclusive; and we assume in computer time calculations that almost all pairs $m \pm 1$ which pass the Fermat tests are twin prime pairs. From these considerations the mean time to locate a TPM can be estimated from $Q(x)$, which is used to derive sieving times and the frequency of required Fermat tests; and from the Hardy-Littlewood estimate, which estimates the probability of a successful pair of Fermat tests. The problem of determining the most efficient sieve limit $x$ is, thus, reduced to a straightforward minimization problem involving the relevant asymptotic formulas.

2. Primality Tests. Once a possible TPM $m$ is located using the sieve and Fermat tests we can test $m+1$ for true primality as follows. If $b$ is an integer such that $b^{m} \equiv 1(\bmod m+1)$ but for every prime factor $q$ of $m$ we have $b^{m / q} \equiv 1$ $(\bmod m+1)$, then $b$ is a primitive root of the prime $m+1$ [5].

The number $m-1$ can be tested using a Lucas-Lehmer test [7]. Let $Q$ be chosen so that $D=1-4 Q$ satisfies $D^{(m-2) / 2} \equiv-1(\bmod m-1)$ and g.c.d. $(m-1, Q D)=1$. Define $U_{0}=0, U_{1}=1$ and for $n \geqslant 2, U_{n}=U_{n-1}-Q U_{n-2}$. If $U_{m} \equiv 0(\bmod m-1)$ but for every prime factor $q$ of $m$ we have $U_{m / q} \not \equiv 0$ (mod $m-1)$, then $m-1$ is prime. We call $Q$ a Lucas-Lehmer parameter for the prime $m-1$.

For these primality tests to be directly applicable it is necessary to know the prime factors of $m$. In the present search a useful factorization algorithm has been Pollard's rho method [8], [9]. The method was used, for example, to find the two ten-digit factors of the second TPM listed in Table 1.

The battery of computer algorithms used in this search is comprised of:

(1) sieve algorithm, sieve limit $x$;

(2) power algorithm $b^{y}(\bmod z)$;

(3) Lucas-Lehmer test $U_{k}(\bmod z)$, parameter $Q$;

(4) Pollard's rho algorithm.

Fermat testing is of, course, a special case of (2), and is assumed to be a process of order $O\left(\log ^{3} z\right)$.

3. Method of Interval-Sieving. This search method finds in principle all TPMs in an interval, though at the expense of necessitating factorization of any suspected TPMs. Choose some large integer $n \equiv 0(\bmod 6)$ as the first member of an arithmetic progression $\{n, n+6, \ldots, n+6(u-1)\}$. We establish a binary array of bits $b(1)$, $\ldots, b(u)$ all set initially to zero and choose a sieve limit $x$. For each prime $p \in P_{x}$ we compute $r^{ \pm}(n, p)$ as the least positive values of $\pm 6^{-1}-1+(n / 6)(\bmod p)$. Then, we set bits $b\left(r^{ \pm}(n, p)+L p\right)=1$ for all nonnegative $L$ such that the array argument does not exceed $u$. The result of this sieve operation is that the only possible TPMs in the progression are of the form $m=n+6(j-1)$ where $b(j)=0$. Approximately 
$u \cdot Q(x)$ numbers $m$ will so survive the sieve, and these $m$ are to be Fermat-tested.

With a sieve limit $x=2000$ the two 22-digit TPMs in Table 1 were found. There are no TPMs lying strictly between these two.

With sieve limit $x=10000$ the 32-digit TPM of the table was found. For numbers of this size and smaller, a small sieve and Pollard rho method in conjunction are generally sufficient for factorization of $m$. On the idea that perhaps 60-digit factorizations are beyond the reach of present techniques [8], it is clear that an alternative search method is required for TPMs having, say, hundreds of digits.

4. A Monte Carlo Method. One of us (M.P.) has refined a search method which circumvents factorization altogether. The idea is to establish an a priori form

$$
m=2 \cdot 3 \cdot s_{1} \cdots s_{j} \cdot q_{1} \cdots q_{k},
$$

where the $s_{i}$ are (possibly) manually chosen small primes, and the primes $q_{i}$ are generated randomly subject to the constraints that $q_{i}<2^{W}$, where $W$ is a machine arithmetic word length; and $m$ is in some predetermined region.

The procedure is to choose a sieve limit $x$ and possible factors $s_{i}$ then to perform these steps:

(1) generate the $q_{i}$ and form $m$;

(2) sieve $m \pm 1$ with all primes in $P_{x}$;

(3) Fermat-test $m+1$;

(4) Fermat-test $m-1$.

On failure at any step, one reverts back to step (1). When a trial $m$ survives all four steps it is a likely TPM, and true primality tests are to follow.

The procedure was programmed on a PDP 11/70 computer with the outer loops written in the language $C$ and the inner loops in assembly language. The word length of the machine is $W=15$; and the basic arithmetic process, a $W$-by- $W$ bit multiply and two adds, consumed 12 microseconds.

A formula was derived for the expected time to find an $m$ which survives all four steps in terms of the rough magnitude of $m$ and the sieve limit $x$. In the case that no $s_{i}$ are chosen, the formula arises as follows. Enumerate the consecutive primes in $P_{x}$ as $p_{1}, \ldots, p_{n}$ and denote for convenience $Q\left(p_{0}\right)=1$. The probability that $p_{k}$ is the smallest prime dividing $m^{2}-1$ is taken to be $\left(2 / p_{k}\right) Q\left(p_{k-1}\right)$. An estimate for the expectation of the number of sieve divisions at step (2), per choice of $m$ in step $(1)$, is

$$
\sum_{k=1}^{n} \frac{2 k}{p_{k}} Q\left(p_{k-1}\right) \sim \frac{A x}{\log ^{4} x},
$$

where $A$ is a constant. The actual time spent for a sieve division of $m \pm 1$ is assumed to be proportional to $\log m$ but essentially independent of $k$, so that the expected time spent at step (2), per choice of $m$ in step (1), is estimated as

$$
\frac{B x \log m}{\log ^{4} x}
$$


for a machine-dependent constant $B$. Since a fraction $Q(x)$ of the $m$ chosen in step (1) will pass step (2), the expected time spent at step (3), per choice of $m$, is estimated as

$$
C Q(x) \log ^{3} m,
$$

where $C$ is another machine constant. The time spent at step (4) is assumed to be negligible relative to that spent at step (3). From the Hardy-Littlewood formula we expect $O\left(\log ^{2} m\right)$ choices of $m$ in step (1) before we find a number which passes all four steps. Such a number will be found, therefore, in an expected time

$$
T=\frac{H x \log ^{3} m}{\log ^{4} x}+\frac{L \log ^{5} m}{\log ^{2} x},
$$

where $H, L$ are machine constants.

A good choice for $x$ which essentially minimizes $T$ is that for which

$$
\pi(x)=(2 L / H) \log ^{2} m .
$$

This implies that the number of primes in the most efficient sieving set $P_{x}$ is proportional to the square of the number of digits in $m$.

With the computer actually used for the search, the ratio $L / H$ was empirically determined to be 0.02 , implying that searching in, say, the 100-digit region should involve an $x$ with $\pi(x)$ about equal to 2100 . Thus, the choice $x=2^{15}, \pi(x)=$ 3512 , an extremely convenient one for the programmer, turned out to be an efficient one as well. The 64, 136, 154, 203, and 303 digit TPMs in Table 1 were found with this method. Though $x=2^{15}$ is highly efficient only for 100 to 150 digits the same value for $x$ was maintained in the searches for the largest TPMs with only a moderate degradation of efficiency.

5. A Statistical Test in the 50-54 Digit Region. A test of the Hardy-Littlewood formula was carried out using 132947 trial values for $m$ in the method of the last section. These values were constrained by $s_{1}=5, s_{2}=7, s_{3}=11, s_{4}=13, q_{i}<$ $2^{15}$, and $10^{49}<m<10^{54}$.

The Hardy-Littlewood formula predicts that such a computer run should produce about

$$
\left(\frac{2 \cdot 3}{Q(13)}\right) \cdot 132947 \cdot \frac{2 C_{2}}{\log ^{2} z}
$$

TPMs, where $z$ is some real number between $10^{49}$ and $10^{54}$. The correction factor $(2 \cdot 3) / Q(13)$ arises from the special form for the trial $m$ values. The correction factor for the less specialized $q_{i}$ constraint is assumed to be negligible. Assuming Poisson statistics for the experimental occurrence of TPMs, this prediction amounts to $245 \pm$ 25 TPMs, with the error corresponding to the ambiguity in $z$ combined with one standard deviation $(245)^{1 / 2}$.

The results of the actual computer run are as follows. Exactly 249 numbers $m$ passed all four steps of the Monte Carlo procedure. Each one of these $m$ was shown to be a TPM in subsequent primality tests. The Monte Carlo procedure consumed a total of five hours C.P.U. time, and the required 498 primality tests took another five hours. 
It is of interest that this statistical test gives an experimental value for $C_{2}$ which is within $2 \%$ of the Hardy-Littlewood prediction.

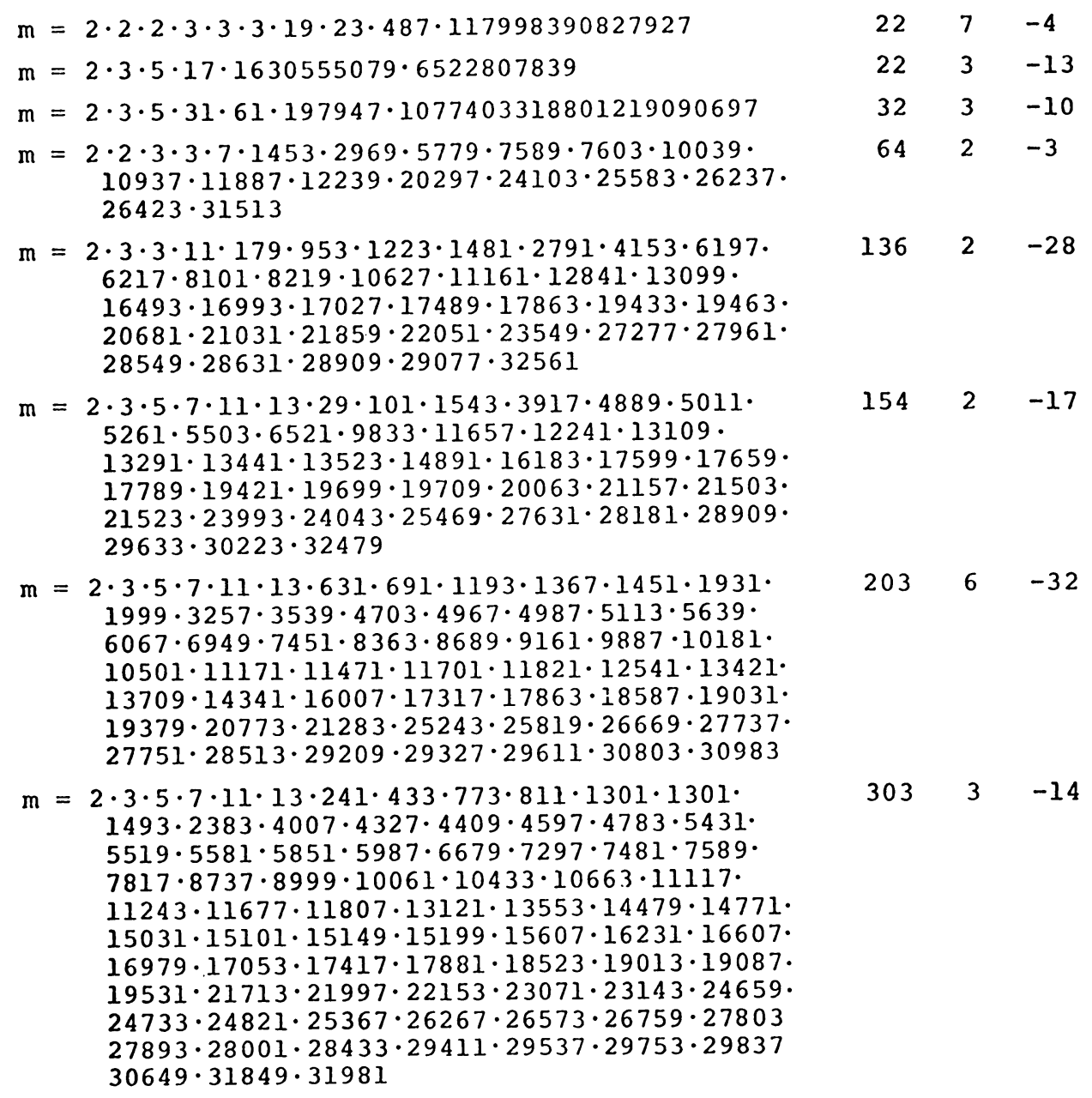

TABLE 1.

Twin primes $m \pm 1$ with primitive roots $b$ for $m+1$; and Lucas-Lehmer parameters $Q$ for $m-1$. All factors listed are primes. The first three $m$ values were found using interval-sieving, so these factorizations required implementation of factoring algorithms. For the remaining five $m$ values, the factors were chosen a priori as explained in the text.

Acknowledgements. The authors wish to thank the administration of Reed College, in whose Computer Center much of this work was performed.

2234 S. E. 20th Avenue

Portland, Oregon 97214

2133 S. E. Clinton Street

Portland, Oregon 97202

1. V. BRUN, "La série $1 / 5+1 / 7+1 / 11+1 / 13+\cdots$ est convergente ou finie," Bull. Sci. Math., v. 43, 1919, pp. 104, 124-128; Chapter XVI. 
2. G. H. HARDY \& J. E. LITTLEWOOD, "Some problems of Partitio Numerorum," Acta Math., v. 44, 1923 , pp. 1-70.

3. D. H. LEHMER, "Tables concerning the distribution of primes up to 37 millions," $M T A C$, v. 13, 1959, pp. 56-57, UMT 3.

4. G. H. HARDY \& E. M. WRIGHT; An Introduction to the Theory of Numbers, 4th ed., Clarendon Press, Oxford, 1965.

5. R. P. BRENT, "Irregularities in the distribution of primes and twin primes," Math. Comp., v. 29,1975 , pp. $43-56$.

6. D. H. LEHMER, "Computer technology applied to the theory of numbers," Studies in Number Theory, vol. 6, W. J. LeVeque (Ed.), Math. Assoc. Amer.; distributed by Prentice-Hall, Englewood Cliffs, N. J., 1969, pp. 117-151.

7. D. E. KNUTH, The Art of Computer Programming, vol. 2, Addison-Wesley, Reading, Mass., 1969; Section 4.3.1, 4.5.4.

8. R. GUY, "How to factor a number," Proc. 5th Manitoba Conference on Numerical Mathematics, Oct. 1-4, 1975 , pp. 49-89. $331-334$.

9. J. M. POLLARD, "A Monte Carlo method for factorization," BIT, v. 1 5, 1975, pp. 